\title{
Autoeficacia de Profesores en Formación Inicial en el Uso de Tecnología para Enseñar Matemáticas
}

\author{
Pre-service Teacher Self-Efficacy in the Use of Technology for Teaching \\ Mathematics
}

\author{
Jaime Andrés Carmona-Mesa* \\ ORCID iD 0000-0002-0100-7090 \\ Difariney González-Gómez ${ }^{* *}$ \\ ORCID iD 0000-0002-0175-2613 \\ Jhony Alexander Villa-Ochoa ${ }^{* * *}$ \\ ORCID iD 0000-0003-2950-1362
}

\begin{abstract}
Resumen
La autoeficacia ha demostrado ser un predictor fuerte de las acciones que los sujetos proyectan realizar para alcanzar un propósito específico. El objetivo del artículo es informar en qué medida se aumenta la autoeficacia de profesores en formación inicial en el uso de tecnología para enseñar matemáticas, a partir de un curso de 64 horas diseñado con características específicas para promover la autoeficacia. La muestra estuvo constituida por dieciséis profesores en formación quienes auto diligenciaron el instrumento de medición de autoeficacia Preparación en el uso de tecnología para enseñar matemáticas, que consta de una batería de 6 dimensiones y con 23 variables ordinales en la escala de 0 a 10 . Se realizaron dos mediciones, la primera al inicio del curso y la segunda después de haberlo realizado. Para el análisis de los datos se aplicaron pruebas no paramétricas, a saber, correlación de Spearman, prueba de Kruskal-Wallis, prueba U de Mann-Whitney y prueba de Wilcoxon. Los resultados indican que la edad, la experiencia profesional y el género no son variables significativas para la autoeficacia de los futuros profesores en el uso de tecnología para enseñar matemáticas. Estadísticamente, se encontraron diferencias significativas entre los rangos de la autoeficacia antes y después de la implementación del curso. Los futuros profesores reportaron sentirse preparados para usar diferentes tecnologías para enseñar matemáticas, sin embargo, consideran necesario tener más experiencias de formación.
\end{abstract}

Palabras clave: Necesidades de formación. Creencias de capacidad. Curso de tecnología. Estudio de caso. Datos y análisis cuantitativos.

\begin{abstract}
Self-efficacy has proved to be a powerful predictor of performances that people plan to attain a specific goal. The purpose of this article is report to what extent self-efficacy of pre-service teachers in the use of technology

\footnotetext{
* Magíster en Educación por la Universidad de Antioquia (UdeA). Profesor en la Universidad de Antioquia, Facultad de Educación, Medellín, Colombia. Dirección postal: Calle 67, n. 53-108, Oficina 9-415, Medellín, Colombia. E-mail: jandres.carmona @udea.edu.co.

** Doctora en Educación por la Universidad de Antioquia (UdeA). Profesora en la Universidad de Antioquia, Facultad Nacional de Salud Pública, Medellín, Colombia. Medellín, Colombia. Dirección postal: Calle 67, n. 53108, Medellín, Colombia E-mail: difariney.gonzalez@udea.edu.co.

*** Doctor en Educación por la Universidad de Antioquia (UdeA). Profesor asociado Universidad de Antioquia, Facultad de Educación, Medellín, Colombia. Dirección postal: Calle 67, n. 53-108, Oficina 9-415, Medellín, Colombia. E-mail: jhony.villa@udea.edu.co.
} 
for teaching mathematics is transformed, during a 64-hour course designed to promote self-efficacy. The sample consisted of sixteen teachers in training who self-completed the self-efficacy measurement instrument "Preparation in the use of technology to teach mathematics" which consists of a battery of 6 dimensions and 23 ordinal variables in the scale of 0 to 10 . Two measurements were carried out, the first one at the beginning and the second one right after the course. Nonparametric tests were performed for data analysis: Spearman's rank correlation coefficient, the Kruskal-Wallis test, the Mann-Whitney U test, and theWilcoxon test. The results indicate age, professional experience, and gender as non-significant variables on pre-service teacher self-efficacy in the use of technology for teaching mathematics. Statistically, there were significant differences between selfefficacy ranges before and after course implementation. Pre-service teachers reported feeling prepared to use technology for teaching. However, they consider necessary to have more training experiences.

Keywords: Training needs. Self-efficacy. Technology course. Case study. Quantitative data and analysis.

\section{Introducción}

Las investigaciones informan que el uso de tecnologías como sistemas de álgebra computacional, software de geometría dinámica y hojas de cálculo en la enseñanza de las matemáticas permiten que los estudiantes logren razonamientos más estructurados en contraste con la formación tradicional (DRIJVERS et al., 2016). Sin embargo, a pesar de registrarse evidencia que donde los futuros profesores tienen gran familiaridad en el uso de este tipo de tecnologías (CARMONA-MESA; ARIAS-SUÁREZ; VILLA-OCHOA, 2019), es necesario ampliar en investigaciones que permitan comprender como trascender de un uso personal a un uso educativo (CARMONA-MESA; SALAZAR; VILLA-OCHOA, 2018).

En investigaciones que analizan la formación de profesores en tecnología, se registran esfuerzos por caracterizar un conjunto de competencias digitales que favorezcan identificar las necesidades y tomar decisiones en la planificación de programas (p. ej. CARVAJAL, 2018), y por promover experiencias en las que sus aprendizajes de la matemática estén mediados por tecnologías, irrumpiendo las prácticas de enseñanza expositivas tradicionales (CASTRILLÓN-YEPES; CARMONA-MESA; VILLA-OCHOA, 2020). Complementario a lo anterior, se registra como una tendencia emergente la indagación por las características de los cursos destinados a formar profesores en el uso de tecnología y sobre las maneras para evidenciar las transformaciones que genera cada una de ellas en las acciones de estos (ABBITT; KLETT, 2007; LEE; LEE, 2014; PARASKEVA; BOUTA; PAPAGIANNI, 2008; ROBERTSON; AL-ZAHRANI, 2012).

$\mathrm{Al}$ respecto, por una parte, en la literatura se registran características significativas en el diseño de cursos específicos (TONDEUR et al., 2012; CARMONA-MESA; VILLAOCHOA, 2017), pero estas no han sido suficientes para aumentar las actitudes de los futuros profesores para el uso de tecnología (CARMONA-MESA; SALAZAR; VILLA-OCHOA, 2018; AGYEI; VOOGT, 2011). En este sentido, es importante explorar nuevos diseños 
curriculares que reflejen las tendencias educativas globales y respondan a las necesidades de formación emergentes en la actualidad (CARMONA-MESA; VILLA-OCHOA, 2017; ROBERTSON; AL-ZAHRANI, 2012).

Por otra parte, para corroborar la pertinencia de tales diseños se debe estudiar cómo y en qué medida logran aumentar la capacidad que creen tener los futuros profesores en el uso de tecnología para enseñar matemáticas (SHAUGHNESSY, 2004). En ese sentido, lograr creencias positivas implica mayor compromiso y persistencia en los procesos de enseñanza (BANDURA, 1994; SHAUGHNESSY, 2004) que trascienden las limitaciones de capacidad tecnológica instalada en las instituciones (AGYEI; VOOGT, 2011), la persistencia de creencias negativas implica obstáculos en el ejercicio profesional (BANDURA, 2006), más probabilidades de expresar frustración y el evitar usar tecnología (DIGREGORIO; LISTON, 2018).

En coherencia con lo anterior, este artículo tiene por objetivo informar en qué medida se aumenta la autoeficacia para usar tecnología al enseñar matemáticas en la formación inicial de profesores. Para alcanzar el objetivo planteado fue necesario, por un lado, diseñar un curso con características específicas para influenciar en el aumento de la autoeficacia y, por otro lado, diseñar un cuestionario que permitió describir la autoeficacia para enseñar matemática a través de tecnologías. Ambos diseños se describen en este artículo, en los apartados 5 y 4 , respectivamente.

\section{Autoeficacia en la formación inicial de profesores}

El término de autoeficacia es teorizado por Bandura (1977), quien lo define como las creencias de las personas sobre su propia capacidad para organizar y ejecutar las acciones necesarias para alcanzar determinados resultados. La atención se centra en los juicios que cada individuo tiene sobre su capacidad y no en las habilidades (TEO, 2009). Por lo tanto, las creencias relativas a la capacidad propia juegan un papel importante en la formación de profesores, en tanto permiten indagar y explicar el efecto de los diseños curriculares a partir del sentimiento de preparación en el uso de tecnología para la enseñanza; además brindan información sobre cómo transforman sus acciones en su práctica profesional (LILJEDAHL; OESTERLE, 2014). En consecuencia, creencias positivas implican mayor planeación, esfuerzo, compromiso y persistencia en los procesos de enseñanza (ABBITT, 2011; BANDURA, 1994; MAMA; HENNESSY, 2013; SHAUGHNESSY, 2004), mientras que creencias negativas implican obstáculos en el ejercicio profesional (BANDURA, 2006; 


\section{DIGREGORIO; LISTON, 2018).}

Las investigaciones que analizan la autoeficacia de los futuros profesores se pueden dividir en tres grupos: estudios que discuten, de forma general, la autoeficacia sin centrarse en las particularidades de alguna disciplina (KILINÇ, 2011); estudios que examinan la autoeficacia en disciplinas específicas, este es el caso de la presente investigación (WILKERSON et. al., 2018); estudios que analizan la autoeficacia en el uso de tecnología, en donde las disciplinas tienden a ser un contexto más que el objeto de investigación (ABBITT, 2011; ABBITT; KLETT, 2007; AL-AWIDI; ALGHAZO, 2012; ALVARENGA, 2011; MAMA; HENNESSY, 2013; SHAUGHNESSY, 2004; TEO, 2009; USHER; PAJARES, 2008).

Un ejemplo que permite ampliar el ultimo conjunto de estudios es la investigación desarrollada por Pamuk y Peker (2009) quienes, si bien analizan la autoeficacia de futuros profesores turco de ciencias y matemáticas, su objetivo se centró en analizar la relación entre la autoeficacia y las actitudes en tecnología al considerar variables como género, nivel de formación y ser propietario de un computador.

Las investigaciones que consideran como objeto de estudio la especificidad temática de la autoeficacia para el uso de tecnología al enseñar matemáticas son escasas. Al respecto, Costa y Assis, (2019) desarrollaron una revisión sistemática de tesis y disertaciones sobre creencias de autoeficacia en educación, alojadas en la base de datos de la Coordinación para Perfeccionar el Personal de la Educación Superior ${ }^{1}$ (CAPES). Estos autores informan que, de los 92 trabajos seleccionados, ocho analizaban la enseñanza de las matemáticas y cinco el uso de tecnología, sin embargo, ninguno de los estudios analizó la autoeficacia para el uso de tecnología al enseñar matemáticas.

En la literatura consultada en la presente investigación, sólo se registra el estudio de Doukakis et al. (2010) en el análisis de la especificidad temática de interés. Sin embargo, Doukakis et al. (2010) informan que el análisis de los datos no mostró valores estadísticos significativos en la autoeficacia para el uso de tecnología luego de la asignatura obligatoria Teaching Mathematics - Practicum Phase. Los investigadores advierten que al parecer los futuros profesores ya habían adquirido los conocimientos necesarios para su uso antes de ingresar a la universidad.

De la anterior investigación se resalta que al considerar la especificidad de la autoeficacia es obligatorio definir la relación teórica y metodológica del constructo que se

\footnotetext{
${ }^{1}$ Traducido del original em Portugués: Coordenação de Aperfeiçoamento de Pessoal de Nível Superior.
} 
articula con ella (BANDURA, 2012); en términos de Fink (2003), es necesario analizar la fiabilidad y validez del instrumento desarrollado. Por ello, una posible causa de los valores no estadísticamente significativos en la investigación de Doukakis et al. (2010) tiene que ver con el contenido de la asignatura obligatoria; es decir, la información metodológica y su relación con el constructo teórico de la autoeficacia. Lamentablemente, el contenido de la asignatura explicitado en la investigación es insuficiente para analizar los objetivos de la intervención y determinar cómo estos influyeron en la autoeficacia.

Detallar el contenido de la asignatura implementada en las investigaciones, además de brindar información para examinar las situaciones desafío que se proponen (BANDURA, 2006) y su relación con el constructo teórico (BANDURA, 2012), es una necesidad reportada por la comunidad académica. Al respecto, Alvarenga (2011) resalta el vacío en estudios que miden la autoeficacia, antes y después de un programa de intervención, que permita verificar: (a) si el programa realmente ha contribuido a un cambio en la creencia de autoeficacia, (b) la relación entre la autoeficacia para el uso de tecnología en tareas personales y usos educativos, y (c) la correlación entre la autoeficacia para enseñar y para usar tecnología en la enseñanza.

Las investigaciones referenciadas en este documento usaron cuestionarios para el registro de la información que son importantes y necesarios, en tanto aportan estrategias significativas para aumentar la autoeficacia de los futuros profesores al usar tecnología, pero no son suficientes para estudiar la autoeficacia en el uso de tecnología para enseñar matemáticas debido a la ausencia de información en el análisis funcional específico (BANDURA, 2006, 2012) e ignoran, en su mayoría, que la autoeficacia de los profesores varía en las diferentes disciplinas (PRIETO, 2005, 2018). Para atender este sesgo en la definición de variables y de clasificación inadecuada de los individuos en las mismas (FINK, 2003; SZKLO; NIETO, 2003), se diseñó el cuestionario Preparación en el uso de tecnología para enseñar matemáticas, que retoma las investigaciones actuales y busca aportar en las dificultades que éstas reportan.

Las anteriores consideraciones ponen en evidencia que, para alcanzar el objetivo propuesto en esta investigación, se era necesario adelantar dos acciones concretas. Por un lado, diseñar un curso con características específicas para influenciar en el aumento de la autoeficacia (sección 5) y, por otro lado, diseñar un cuestionario que permitiera determinar por medio de un puntaje la autoeficacia para enseñar matemática a través de tecnologías (sección 4). 


\section{Método}

Esta investigación es un estudio de caso con datos y análisis cuantitativos (GILLHAM, 2000) que indaga la autoeficacia de dieciséis profesores de matemáticas en uno de los siete programas de Licenciatura en Matemáticas acreditados por el Ministerio de Educación Nacional de Colombia. La muestra fue a conveniencia por las características regulares del tipo de curso analizado (SZKLO; NIETO, 2003), que normalmente oscilan, en todos los programas, entre 10 y 25 estudiantes matriculados.

Se realizó un estudio cuasi-experimental pre-test / post-test para proporcionar información cuantitativa de tendencias o actitudes de una población mediante el estudio de una muestra. Este tipo de estudios permiten al investigador la generalización de resultados a la población objeto de estudio, obtenidos mediante muestras pequeñas sin la necesidad de manipular o controlar variables (CRESWELL, 2014). Por lo tanto, existe un control sobre el factor de estudio que, en contexto de la autoeficacia, permite confirmar o negar el incremento de la autoeficacia una vez finalizado el programa de intervención.

El cuestionario fue auto diligenciado anónimamente por los profesores en formación inicial y nunca representó una valoración en el seguimiento del curso. El diseño del cuestionario sigue las sugerencias de Fink (2003) y Bandura $(2006,2012)$ en fiabilidad y validez que le da rigor a la investigación. Además, se complementan los análisis con una descripción meticulosa de la intervención que favorece un impacto mayor de la misma (GILLHAM, 2000).

\section{Cuestionario Preparación en el uso de tecnología para enseñar matemáticas}

El diseño de un cuestionario que proporcione información sobre la autoeficacia en el uso de tecnología para enseñar matemáticas implica una definición clara de una perspectiva teórica, los descriptores a utilizar para medirla, la manera de redactar y de enunciar, de forma exhaustiva, dichos descriptores o ítems y, finalmente, la organización y presentación del instrumento con el fin de medir las creencias de la capacidad propia en el dominio de la actividad específica (FINK, 2003).

En primer lugar, Bandura (1994) señala que la autoeficacia permite evaluar cuatro tipos de procesos, a saber: posibles escenarios que resultarán de las acciones antes de actuar y plantear sus metas - proceso cognitivo -; expectativas de resultados, atribuciones causales y metas cognitivas - proceso de motivación -; depresión, niveles de ansiedad y activación del 
arousal - proceso afectivo -; selección de los ambientes y actividades donde se consideran hábiles - selectivo.

En segundo lugar, en relación con los factores que influencian la autoeficacia, Bandura (1994) plantea cuatro: experiencias de dominio, experiencia vicaria, persuasión verbal y estado fisiológico. Las experiencias positivas en situaciones que implican desafíos son más efectivas para crear un fuerte sentido de dominio real - experiencia de dominio -; observar a otras personas ejecutar de forma exitosa en ciertas actividades permite crear y fortalecer creencias de capacidad para enfrentar diferentes desafíos - experiencia vicaria -; persuadir verbalmente a las personas de que poseen las capacidades necesarias para dominar diferentes actividades con éxito es la forma más difícil de influenciar - persuasión verbal -; por último, los estados de ánimo, emociones, reacciones físicas y los niveles de estrés pueden repercutir en cómo una persona se siente respecto a sus habilidades personales, indicadores como cansancio o nervios afectan, de forma significativa, la realización de tareas - estado fisiológico.

En tercer lugar, para medir la autoeficacia, en el diseño de los cuestionarios es necesario considerar la formulación de las afirmaciones en términos de juicios de capacidad vinculados a situaciones que plantean desafíos - nivel de dificultad - y se espera un desempeño exitoso (BANDURA, 2006). Los cuestionarios, bajo una escala Likert, son los de mayor uso para medir las creencias en la capacidad propia (ABBITT, 2011; ABBITT; KLETT, 2007; BANDURA, 2006; TEO, 2009); la escala para analizar autoeficacia inicia en cero e incluye un nivel de medición amplio para evitar imprecisión en la misma; de acuerdo a Bandura (2006, 2012), escalas con puntajes de 0 a 10 son más sensibles y confiables, psicométricamente, que otras con menos ítems.

En consecuencia a lo anterior, se definió en el cuestionario Preparación en el uso de tecnología para enseñar matemáticas una escala de 25 ítems, con un puntaje para cada uno de 0 a 10, a saber: (0) No me siento preparado, (5) Me siento relativamente preparado y (10) Me siento altamente preparado. Los 25 ítems cuentan con validez de apariencia realizada por expertos (FINK, 2003), a los cuales se les solicitó juzgar cada ítem en categorías de suficiencia, claridad, coherencia y relevancia (ESCOBAR-PÉREZ; CUERVO-MARTÍNEZ, 2008). 


\subsection{Redacción de los ítems del cuestionario Preparación en el uso de tecnología para enseñar matemáticas}

Para la redacción de los ítems y para la validez de contenido en relación a los procesos que se pueden evaluar a partir de la autoeficacia (FINK, 2003), se revisaron las publicaciones de Bandura (1977, 1994, 2006, 2012) y se utilizaron varias preguntas del instrumento Computer Technology Integration Survey - CTI, los ítems seleccionados fueron discutidos por un grupo de expertos y adaptados al contexto. El CTIS fue creado y validado por Wang, Ertmer y Newby (2004) y en investigaciones posteriores Abbitt (2011), Abbitt y Klett, (2007) y Al-Awidi y Alghazo (2012) lo han utilizado.

En el desarrollo del cuestionario Preparación en el uso de tecnología para enseñar matemáticas, inicialmente se presentó un cuestionario que contenía treinta ítems y, con este, se realizaron dos pruebas piloto con el fin de evaluar para cada ítem la claridad y la coherencia en su escritura. La primera prueba piloto se aplicó a diez futuros profesores de diferentes disciplinas. La segunda se realizó a veinte estudiantes de un programa de formación para futuros profesores en matemáticas. Los datos obtenidos permitieron identificar la información medida en relación a la actividad de dominio específico. Como resultado de estos dos estudios, la redacción de los ítems se depuró, algunos se eliminaron y se redactaron otros indicadores emergentes. Se resaltan los ítems 24 y 25 porque se desarrollaron para contrastar y verificar la consistencia de las creencias de capacidad informadas en los anteriores ítems (FINK, 2003; SZKLO; NIETO, 2003; MAMA; HENNESSY, 2013).

El ítem 24 indaga por las creencias de capacidad para utilizar software y hardware para enseñar matemáticas, y presenta catorce subítems que indagan sobre las tecnologías exploradas en el curso. Por otra parte, el ítem 25 cuestiona las creencias de capacidad para utilizar tecnología al enseñar temáticas específicas de las matemáticas y presenta diez subítems. Veamos el Cuadro 1.

1. Estoy preparado para solucionar diferentes dificultades en la manipulación de tecnologías.

2. Estoy preparado para utilizar las experiencias previas frente al uso de tecnología, como ayuda para manipular las tecnologías propuestas en el Seminario de Especialización II.

3. Estoy preparado para usar la terminología correcta al enseñar matemáticas con tecnología.

4. Estoy preparado para aprender autónomamente a integrar tecnología en la enseñanza de las matemáticas.

5. Estoy preparado para utilizar diferentes tecnologías en la clase de matemáticas.

6. Estoy preparado para que, con el tiempo, mi capacidad para hacer frente a las necesidades tecnológicas de los estudiantes mejore.

7. Estoy preparado para actualizarme en el uso de las tecnologías que integro en clases de matemáticas.

8. Estoy preparado para reconocer las experiencias de otros colegas como referentes en la planeación de mis clases.

9. Estoy preparado para utilizar la tecnología como medio para transformar la sociedad, permeada por intereses políticos, económicos y culturales. 
10. Estoy preparado para identificar las potencialidades del uso de diferentes tecnologías en la enseñanza de las matemáticas.

11. Estoy preparado para identificar las fortalezas del uso de diferentes tecnologías en la enseñanza de las matemáticas.

12. Estoy preparado para identificar las debilidades del uso de diferentes tecnologías en la enseñanza de las matemáticas.

13. Estoy preparado para reconocer el componente didáctico al momento de usar cualquier tecnología en la clase de matemáticas.

14. Estoy preparado para reconocer el componente pedagógico de usar cualquier tecnología en la clase de matemáticas.

15. Estoy preparado para reconocer el componente matemático al momento de usar cualquier tecnología en la clase de matemáticas.

16. Estoy preparado para diseñar mi clase de matemáticas donde los estudiantes utilicen la tecnología en el aprendizaje de conceptos matemáticos.

17. Estoy preparado para adaptar experiencias que integran recursos tecnológicos y promueven el aprendizaje en los estudiantes.

18. Estoy preparado para evaluar el conocimiento matemático adquirido por mis estudiantes al usar tecnología en su proceso de aprendizaje.

19. Estoy preparado para afrontar el uso de la tecnología en la clase de matemáticas con la infraestructura que se dispone en Colombia.

20. Estoy preparado para identificar las necesidades de los estudiantes al adquirir los conocimientos matemáticos con el uso de tecnología.

21. A partir de las experiencias logradas en el curso, considero que tengo más habilidades frente al uso de la tecnología en la clase de matemáticas.

22. Al observar el uso de tecnología por mis compañeros del Seminario de Especialización II, me considero más preparado para usarla.

23. Estoy preparado para usar tecnología al enseñar matemáticas, de forma similar o mejor, a mis profesores del Seminario de Especialización II.

24. Preparación para usar tecnología (se presentan 14 sub ítems sobre tecnologías - Calculadora simple, TI Voyage, GeoGebra, Modellus, Tracker, Sensores, Multímetro, Internet, OVA, Powtoon, Cabri, Webques, Facebook y Excel- con la misma escala de 0 a 10).

25. Preparación para usar tecnología al enseñar matemáticas (se presentan 10 contenidos matemáticos Aritmética, Geometría, Geometría analítica, Estadística, Probabilidad, Álgebra, Álgebra lineal, Trigonometría, Lógica y Cálculo- con la misma escala de 0 a 10).

Cuadro 1 - Ítems del cuestionario preparación para usar tecnología al enseñar matemáticas Fuente: elaborado por los autores

Observamos que, valores altos en los ítems 1, 2, 4, 6, 7, 9, 17 y 22 (Cuadro 1), deben conservar relación con el promedio de los valores en el ítem 24. En la misma lógica, valores altos en los ítems 3, 5, 10, 11, 12, 13, 14, 15, 16, 18, 19, 20, 21 y 23 (Cuadro 1) deben conservar relación con el promedio de los valores en el ítem 25.

Este nuevo conjunto de 25 ítems (Cuadro 1) fue evaluado por cinco expertos, entre los cuales estuvieron incluidos profesores de matemáticas en la formación inicial, investigadores en el uso de tecnología para la enseñanza de las matemáticas y expertos en estadística. A cada experto se le solicitó juzgar cada ítem en las categorías propuestas (suficiencia, claridad, coherencia y relevancia) y, posteriormente, realizar las observaciones y sugerencias convenientes para mejorar los reactivos (ESCOBAR-PÉREZ; CUERVO-MARTÍNEZ, 2008).

Para fortalecer los niveles descriptivos del estudio y aportar evidencia empírica sobre el dominio funcional específico en la enseñanza de las matemáticas, se garantizó que la redacción de los primeros 23 ítems correspondiera con la naturaleza de las necesidades de 
formación en el uso de tecnología. Esta noción es una adjetivación de seis tipos de necesidades identificadas, por medio de una revisión de literatura (CARMONA-MESA; VILLA-OCHOA, 2017).

\subsection{Necesidades de formación en el uso de tecnología para enseñar matemáticas}

Un limitador del nivel explicativo y predictivo en los ítems de los cuestionarios basados en la autoeficacia es que presentan poca o ninguna relevancia en el dominio en la actividad que está siendo estudiada (BANDURA, 2006, 2012). En la presente investigación, este dominio se centra en el uso de tecnología para enseñar matemáticas y se analiza a partir de necesidades de formación en el uso de tecnología que tanto la literatura como los futuros profesores ponen en evidencia en los cursos de formación. Carmona-Mesa y Villa-Ochoa (2017) identificaron en la literatura internacional seis tipologías con respecto a tales necesidades. Los autores las denominan como: experiencial (ítems 21 al 23), funcional (ítems 1 al 4), actitudinal (ítems 5), ética (ítems 6 al 9), crítica (ítems 10 al 15) y pragmática (ítems 16 al 20).

Conforme Carmona-Mesa y Villa-Ochoa (2017) describieron, las necesidades de formación se adjetivan por la naturaleza de su contenido, a saber: el tipo de estrategia es significativo para movilizar los futuros profesores en la tipología de las necesidades de formación - Experiencial -; considerar las experiencias previas y espacios extracurriculares para una formación técnica - Funcional -motivar un cambio en las creencias, actitudes y confianza en el uso de tecnología - Actitudinal -; considerar cuestiones morales y éticas de la tecnología que son permeadas por asuntos políticos, económicos y culturales - Ética -; develar las fortalezas y debilidades de la tecnología para su uso en la ejercicio profesional Crítica -; desarrollar trabajo de campo en contextos reales previos al ejercicio profesional Pragmática.

\section{Diseño del curso}

El curso era obligatorio en el plan de estudios y orientado al conocimiento del profesor para el uso de tecnologías en la enseñanza de las matemáticas. En su diseño, las necesidades de formación en el uso de tecnología se articularon con los factores que influyen en el desarrollo de la autoeficacia: experiencia de dominio, experiencia vicaria, persuasión verbal y estado fisiológico. Además, se consideraron las estrategias registradas en la literatura como 
experiencias significativas en el diseño de cursos específicos para formar futuros profesores en el uso de tecnología (CARMONA-MESA; VILLA-OCHOA, 2017; TONDEUR et al., 2012).

El curso se desarrolló con una intensidad de 64 horas, periodo de latencia que se corresponde con el implementado en otras investigaciones (p. ej. ABBITT; KLETT, 2007), divididas en sesiones de cuatro horas por semana. La estructura de cada sesión constó de tres momentos: presentación de un artículo de investigación que refería al uso de tecnología, elegido y presentado por un estudiante diferente cada semana; desarrollo de un taller acerca de la producción de conocimientos a través de la tecnología; otra presentación acerca de lecturas que ampliaban el horizonte de los estudiantes en relación con la literatura y las matemáticas, asimismo, propiciaba reflexiones acerca del contenido matemático.

Los talleres se plantearon con el objetivo de motivar el trabajo individual y colectivo, actuaciones en relación con la tecnología y reflexiones en torno a los aprendizajes matemáticos y el rol de los medios en esos aprendizajes. Cada taller se estructuró en tres momentos, en el primero de ellos, se propusieron situaciones, problemas o tareas que los estudiantes debían resolver a través del uso de tecnología. En el segundo momento, se realizó una plenaria en la que cada grupo de estudiantes, expuso sus desarrollos, construcciones, dificultades, y aprendizajes. En caso de requerirse ajustes, los estudiantes podían volver a trabajar en grupos. Finalmente, se promovió la reflexión sobre el rol de la tecnología en la solución de la situación, tarea o problema; también se posibilitó el espacio para la discusión sobre las diferencias y posibilidades de desarrollar las tareas con otras tecnologías y la generación de reflexiones y proyección de posibles prácticas en su futuro desempeño profesional.

Los talleres permitieron experiencias en situaciones que implican desafíos en el desarrollo de los cuatro ejes temáticos: se inició con el uso de la calculadora, luego se analizó software educativo, posteriormente se discutió cómo la tecnología apoya los procesos modelación matemática, y se concluyó con internet, dispositivos móviles y videojuegos y su relación con Educación Matemática. Algunos ejemplos de los talleres y ambiente de clase se pueden encontrar en Carmona-Mesa, Salazar y Villa-Ochoa (2018).

El contenido de los ejes temáticos, para favorecer la persuasión verbal de forma directa (por los orientadores del curso) e indirecta (por la propia experiencia de los futuros profesores), se planteó en un nivel de complejidad tecnológico progresivo en donde los futuros profesores, para cada encuentro, enfrentaban situaciones que les exigían retomar los conocimientos adquiridos en experiencias previas para resolver nuevos problemas y explorar 
nuevas tecnologías.

En los ejes temáticos se reconoció la necesidad de conocimientos en diferentes dominios - pedagógico, tecnológico, didáctico y matemático - que se articularon a las formas de hacerlos y producirlos. Además, como se indicó anteriormente, se reconoció una formación que trascendió el desarrollo experiencias de dominio - individuales y grupales - hacia la reflexión y la proyección de la capacidad para usar tecnologías en el ejercicio profesional, que ponía en evidencia fortalezas, temores y preferencias personales.

\section{Resultados}

Para el registro y fiabilidad de los datos (FINK, 2003), el pre-test se implementó durante el segundo encuentro del semestre y el pos-test en el último encuentro, ambos instrumentos fueron diligenciados por los dieciséis profesores en formación inicial (nueve hombres y siete mujeres). A continuación, se presenta el análisis de los datos por medio del IBM SPSS Statistics 24.

Para establecer las creencias de capacidad de los futuros profesores en el uso de tecnología para enseñar matemáticas, se trabajó con la media de la autoeficacia en los registros pre y post-test. Se realizó una prueba de rangos de Wilcoxon dado el tamaño de muestra tan reducido y la no normalidad en los datos. Esta técnica estadística no paramétrica se usa para comparar el rango medio de dos muestras relacionadas y determinar si existen diferencias entre los puntajes medianos de autoeficacia, antes y después de la implementación del curso específico.

Los resultados informan una diferencia significativa de la autoeficacia de los futuros profesores antes $(\mathrm{M}=4,92 ; \mathrm{DT}=1,199)$ y después $(\mathrm{M}=6,96 ; \mathrm{DT}=1,357)$ del curso $(p=$ 0,001, prueba de rangos de Wilcoxon). Es decir, el puntaje mediano de autoeficacia es diferente antes y después de la intervención. Lo que sugiere que el diseño metodológico del curso y su desarrollo tuvieron efectos significativos en el puntaje de autoeficacia de los futuros profesores en el uso de tecnología para enseñar matemáticas.

Los ítems 24 y 25 se construyeron con el objetivo de contrastar y analizar la consistencia de las creencias de capacidad informadas en los otros ítems. En ese sentido, se definieron como variables aleatorias ordinales las medias para cada ítem, en los registros pre y post (valores subrayados en la Tabla 1) y se halló el coeficiente de correlación de Spearman. En la Tabla 1 se identifica que la relación entre la media del uso de diferentes tecnologías (ítem 24) y las medias de los demás ítems aumenta de forma significativa en el registro post; 
la mayoría de las correlaciones se encuentran en rango alto, excepto los ítems 1, 3, 9, 14, 15 y 19 (valores en negrilla). Los valores de las correlaciones en las medias de los ítems 1 y 9, en relación con la media del ítem 24 , sugieren que la formación técnica y la preparación para utilizar tecnología en situaciones contextuales auténticas no presentan relación con las creencias de capacidad para usar diferentes tecnologías en el ejercicio profesional (Cuadro 1).

Tabla 1 - Correlación de Spearman entre los ítems, uso de tecnologías

\begin{tabular}{|c|c|c|c|c|c|c|}
\hline \multirow[t]{2}{*}{$\begin{array}{l}\text { Necesidades } \\
\text { de formación }\end{array}$} & \multirow{2}{*}{$\begin{array}{c}\text { Ítems (IT) } \\
\text { IT25 }\end{array}$} & \multirow{25}{*}{ 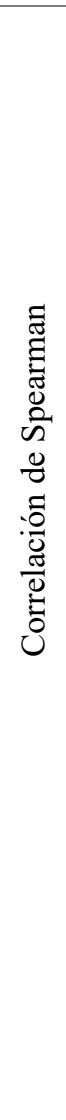 } & \multicolumn{2}{|c|}{$\begin{array}{c}\text { Uso de Tecnologías } \\
\text { (ítem 24) }\end{array}$} & & \multirow[t]{2}{*}{$\begin{array}{c}\text { Diferentes temas } \\
\text { (ítem 25) }\end{array}$} \\
\hline & & &, $521 *$ & $645^{* *}$ & & \\
\hline \multirow{4}{*}{ Funcional } & IT1 & &,- 217 & $\mathbf{3 8 4}$ &, $620^{*}$ &, 361 \\
\hline & IT2 & &,- 108 & $632^{* *}$ &, 367 &, $500^{*}$ \\
\hline & IT3 & &,- 194 &, $\mathbf{3 1 9}$ & ,461 &, $553^{*}$ \\
\hline & IT4 & &, 044 &, $527^{* *}$ &,- 027 & $563^{*}$ \\
\hline Actitudinal & IT5 & & ,217 & $632^{* *}$ & ,341 & 395 \\
\hline \multirow{4}{*}{ Ética } & IT6 & &, 187 &, $583^{* *}$ &, 054 &, $574^{*}$ \\
\hline & IT7 & &,- 251 &, $578^{* *}$ &, 017 &, $528^{*}$ \\
\hline & IT8 & &, 114 &, $647^{*}$ &, 038 &, $697^{*}$ \\
\hline & IT9 & &, 450 &, 432 &, $569^{*}$ &, 323 \\
\hline \multirow{6}{*}{ Crítica } & IT10 & & ,473 &, 454 &, 402 &, 198 \\
\hline & IT11 & &, 478 &, 486 &, 420 &, 187 \\
\hline & IT12 & &, 434 &, $651^{* * *}$ &, 419 &, 304 \\
\hline & IT13 & &, 315 &, $599^{*}$ &, 344 &, $596^{*}$ \\
\hline & IT14 & &, 231 &, 379 &, 344 &, $616^{*}$ \\
\hline & IT15 & & 253 &, 290 & ,297 &, 364 \\
\hline \multirow{5}{*}{ Pragmática } & IT16 & &, $625^{* *}$ & $649^{* * *}$ &, $549^{*}$ &, 429 \\
\hline & IT17 & &, $622^{*}$ &, $589^{*}$ &, 459 &, 294 \\
\hline & IT18 & & ,292 &, $643^{* *}$ &, 356 & 294 \\
\hline & IT19 & &, 495 &, $\mathbf{3 9 9}$ &, $582^{*}$ &, 076 \\
\hline & IT20 & &, $540^{*}$ & $528^{*}$ &, $668^{* *}$ & .270 \\
\hline \multirow{3}{*}{ Experiencial } & IT21 & & ,302 &, $768^{* * *}$ &, 351 &, 491 \\
\hline & IT22 & &, 403 &, $562^{*}$ &, 462 &, $508^{*}$ \\
\hline & IT23 & &, $614^{*}$ &, $503^{*}$ &, $673^{* *}$ &, 025 \\
\hline
\end{tabular}

Fuente: elaborado por los autores

Por otra parte, la media de usar tecnología al enseñar diferentes contenidos matemáticos (ítem 25) y las medias de otros ítems mostraron un aumento a valores de correlación altos, de correlaciones altas y moderadas disminuyen a bajas y muy bajas para los ítems 1, 9, 19, 20 y 23 (valores en negrilla y cursiva de la Tabla 1). Las correlaciones propuestas como contraste entre las medias de los ítems 19, 20 y 23 en relación con la media del ítem 25 (Tabla 1), indican que las creencias de capacidad para usar tecnología en diferentes temáticas no están relacionadas con la infraestructura dispuestas en las aulas colombianas, con la dificultad para identificar las necesidades de los estudiantes para adquirir el conocimiento y por el aprendizaje vicario a partir de las prácticas de los orientadores del 
curso (Cuadro 1).

La correlación entre las medias de los ítems 24 y 25 aumenta el coeficiente de moderado a alto, lo cual sugiere que las creencias de capacidad para usar tecnologías están influenciadas por los contenidos y metodología del curso. En este sentido, por ejemplo, la autoeficacia para usar el software GeoGebra se asocia a la autoeficacia para enseñar Geometría (ítems 24 y 25 en Cuadro 1). Además, las correlaciones en el registro post entre el ítem 24 con los ítems 2, 4, 7, 17 y 22 y el ítem 25 con los ítems 3, 13 y 14, informan que existe mayor consistencia en la información que reportaron los futuros profesores en el uso de diferentes tecnologías que en el uso de tecnología para enseñar diferentes temáticas.

Tabla 2 - Mediana por ítem y grupo de ítems en cada necesidad de formación (pre/post)

\begin{tabular}{|c|c|c|c|c|c|}
\hline $\begin{array}{l}\text { Necesidades de } \\
\text { formación }\end{array}$ & Ítems (IT) & \multicolumn{2}{|c|}{$\begin{array}{l}\text { Mediana } \\
\text { (pre/post) }\end{array}$} & \multicolumn{2}{|c|}{$\begin{array}{c}\text { Mediana por necesidades } \\
\text { (pre/post) }\end{array}$} \\
\hline \multirow{4}{*}{ Funcional } & IT1 & 5 & 7 & \multirow{4}{*}{5} & \multirow{4}{*}{$\underline{8}$} \\
\hline & IT2 & 5 & $\underline{8}$ & & \\
\hline & IT3 & 5 & $\underline{8}$ & & \\
\hline & $\mathrm{T} 4$ & 8 & $\overline{8}$ & & \\
\hline Actitudinal & IT5 & 6 & $\overline{8}$ & 6 & $\underline{8}$ \\
\hline \multirow{4}{*}{ Ética } & IT6 & 9 & $\underline{8}$ & \multirow{4}{*}{9} & \multirow{4}{*}{$\underline{9}$} \\
\hline & IT7 & 9 & $\overline{9}$ & & \\
\hline & IT8 & 8 & $\underline{10}$ & & \\
\hline & IT9 & 6 & $\overline{8}$ & & \\
\hline \multirow{6}{*}{ Crítica } & IT10 & 6 & $\underline{8}$ & \multirow{6}{*}{7} & \multirow{6}{*}{$\underline{8}$} \\
\hline & IT11 & 7 & $\underline{8}$ & & \\
\hline & IT12 & 7 & $\underline{8}$ & & \\
\hline & IT13 & 7 & $\underline{8}$ & & \\
\hline & IT14 & 7 & $\overline{8}$ & & \\
\hline & IT15 & 7 & $\overline{7}$ & & \\
\hline \multirow{5}{*}{ Pragmática } & IT16 & 6 & $\underline{8}$ & \multirow{5}{*}{6} & \multirow{5}{*}{$\underline{8}$} \\
\hline & IT17 & 6 & $\underline{8}$ & & \\
\hline & IT18 & 7 & $\underline{8}$ & & \\
\hline & IT19 & 5 & $\underline{8}$ & & \\
\hline & IT20 & 6 & $\underline{8}$ & & \\
\hline \multirow{3}{*}{ Experiencial } & IT21 & 6 & $\underline{9}$ & \multirow{3}{*}{6} & \multirow{3}{*}{$\underline{8}$} \\
\hline & IT22 & 6 & $\underline{8}$ & & \\
\hline & IT23 & 5 & $\underline{7}$ & & \\
\hline \multirow{2}{*}{ Ítems de contraste } & IT24 & 1 & $\underline{6}$ & 1 & $\underline{6}$ \\
\hline & IT25 & 5 & $\underline{8}$ & 5 & $\underline{8}$ \\
\hline
\end{tabular}

El análisis de los ítems no solo se realizó para cada informante, también se hallaron las medianas por ítem y por grupo de ítems que atendían a la naturaleza de cada necesidad de formación. La Tabla 2 presenta las medianas tanto para el registro pre como el post (valores subrayados); en la mayoría de los ítems se aumentan los valores en el registro post, solo los ítems 4, 7 y 15 conservan el mismo valor. El ítem 6 es el único que presenta una disminución en el valor mediano, esto es, puede interpretarse como un indicador de la pertinencia de actualizar los conocimientos para atender a las necesidades tecnológicas que exigen en esta 
época los estudiantes.

En las medianas por ítems solo el 8 y 24 registran valores extremos (valores en negrilla), esto sugiere, por un lado, que las experiencias de los futuros profesores en el uso de tecnología son significativas para mejorar las creencias de capacidad y, por el otro, ratifica que el diseño metodológico del curso transformó las creencias de capacidad para usar diferentes tecnologías al enseñar matemáticas. Además de los 25 ítems, el cuestionario contenía información sociodemográfica como la edad (edad mínima y máxima 20 y 38 respectivamente, mediana de 25 años), experiencia profesional (dos futuros profesores con ninguna, diez con práctica previa al ejercicio profesional y cuarto profesor en ejercicio) y género ( $56 \%$ masculino y $44 \%$ femenino). Para analizar la relación entre las variables género, la edad y la experiencia profesional con el puntaje de autoeficacia de los futuros profesores en el uso de tecnología para enseñar matemáticas se realizaron pruebas no paramétricas en muestras independientes (U de Mann-Whitney, prueba de Kruskal-Wallis y Correlación de Spearman) dado el tamaño de muestra y el no cumplimiento del supuesto de normalidad.

Los resultados muestran evidencia estadística para concluir que no existen diferencias entre la autoeficacia para usar tecnología al enseñar matemáticas y el género $(p=0,560$, prueba u de Mann-Whitney), es decir que el puntaje mediano de autoeficacia para enseñar matemáticas es el mismo en hombres que en mujeres. Con respecto la edad se encontró correlación ( $p=0,441$, Correlación de Spearman), finalmente no se encontraron diferencias en el puntaje mediano de autoeficacia según las categorías de la variable que recoge la experiencia profesional de los participantes $(p=0,569$, prueba de Kruskal-Wallis). Por lo tanto, de acuerdo a la evidencia estadística el puntaje de la autoeficacia de los futuros profesores para usar tecnología al enseñar matemáticas no presenta diferencias según el género, la edad y la experiencia profesional.

\section{Discusión}

\subsection{Autoeficacia de profesores en formación inicial para usar tecnología al enseñar matemáticas}

Los resultados de esta investigación aportan evidencia estadística sobre la autoeficacia que configuraron los profesores en formación inicial para usar tecnología al enseñar matemáticas a partir de las experiencias logradas en un curso de 64 horas. Además, la evidencia corroboró la necesidad de establecer ítems de contraste, en el desarrollo del 
cuestionario, para mejorar el análisis de las creencias de capacidad auto informadas por los futuros profesores (MAMA; HENNESSY, 2013).

Por una parte, en los registros pre del cuestionario se observó sesgos en el auto informe de la capacidad percibida por los futuros profesores para usar tecnología en el ejercicio profesional, información corroborada por los ítems 24 y 25. Por otra parte, en el registro post del cuestionario, luego del desarrollo del curso, los valores de correlación entre las medias de los ítems que describen la naturaleza de las necesidades de formación y los ítems 24 y 25, informan consistencia en la capacidad percibida por los futuros profesores para usar tecnología al enseñar matemáticas.

De este estudio se derivan cinco resultados importantes. En primer lugar, se presenta evidencia estadística de como el diseño del curso aumentó la autoeficacia y el cuestionario permitió medir dicho aumento de manera eficiente, al considerar el dominio funcional específico a partir de las necesidades de formación en el uso de tecnología. Lo anterior se contrasta con el estudio de Doukakis et al. (2010), en el cual no se encontró, en una muestra 25 futuros profesores, una mejora en la autoeficacia en el uso de tecnología luego de la implementación de un curso destinado a aumentarla. Por lo tanto, el reconocer la especificidad de la autoeficacia en profesores de matemáticas permitió evidenciar información estadística para el análisis funcional específico (BANDURA, 2006, 2012; PRIETO, 2005, 2018), al definir la relación teórica y metodológica tanto para el curso como el cuestionario (FINK, 2003).

En segundo lugar, se brindó evidencia estadística que informa la autoeficacia de los futuros profesores es más fuerte en el uso de diferentes tecnologías que en el uso de tecnología en diferentes temáticas; este resultado sugiere que en los cursos se debe diversificar la integración de tecnología según la temática y evitar sesgos al concentrar las discusiones en solo una. En tercer lugar, la evidencia corroboró lo informado por Siddiq y Scherer (2016), quienes hallaron las creencias de capacidad en el uso de tecnología con fines educativos, como prerrequisito para iniciar reflexiones más elaboradas en sus usos pedagógicos e integración en actividades del aula.

En cuarto lugar, se señala la necesidad de apoyar, desde las instituciones, la formación de sus futuros profesores en cursos específicos que trasciendan la adquisición de habilidades técnicas para la manipulación de recursos tecnológicos a una integración eficiente y consciente en los procesos educativos (ABBITT; KLETT, 2007; KIILI, 2016; SIDDIQ; SCHERER, 2016). Este resultado se corresponde con los planteamientos de Byker et al. (2018), quienes informan los cursos que simultáneamente discuten el conocimiento en 
diferentes dominios - pedagógico, tecnológico, didáctico y matemático - como alternativas más potentes para mejorar la autoeficacia de los futuros profesores al responder a los cambios en la tecnología, y pueden participar activamente en la práctica reflexiva. Estos tres resultados amplían la comprensión frente a los aspectos que intervienen en un curso que se propone aumentar las creencias de capacitada en el uso de tecnología al enseñar (ALVARENGA, 2011).

Finalmente, en quinto lugar se encontró que variables como la experiencia profesional, el género y la edad no influyen en la autoeficacia de los futuros profesores. En cuanto a la experiencia profesional, se ofrece evidencia estadística que contrasta con la investigación de Paraskeva, Bouta y Papagianni (2008), quienes informan que profesores de áreas como matemáticas requieren de experiencias previas para aumentar su autoeficacia.

En relación al género, los resultados corresponden con Pamuk y Peker (2009) y difieren de lo informado por Zhou y Xu (2007), estos últimos presentan evidencia estadística donde los hombres tienen mayor autoeficacia que las mujeres. Con respecto a la edad, se discrepan los indicios de Siddiq y Scherer (2016) al informar que la autoeficacia en el uso de tecnología no se distribuye por igual entre hombres y mujeres, y entre grupos de edad. Esta evidencia se interpreta como producto de dos aspectos: los estilos de aprendizaje particulares de los profesores en el contexto colombiano (DIGREGORIO; LISTON, 2018) y que el diseño metodológico del curso permitió aumentar la autoeficacia de los futuros profesores, sin importar sus características individuales y las experiencias previas en el uso de tecnología y en ejercicio profesional.

\subsection{Aspectos a fortalecer en futuras investigaciones}

Los valores del ítem 15 no tienen cambios significativos en las medias de la autoeficacia en la implementación pre y post, este indicador refiere a la capacidad para identificar el contenido matemático en el uso de tecnología, esto se fundamenta en los coeficientes de correlación en las medias de los ítems 3, 13 y 14 en relación con la media del ítem 25. Por lo tanto, es necesario fortalecer las experiencias que tienen los futuros profesores para aumentar la autoeficacia en el uso de tecnología para enseñar matemáticas.

Zhou y Xu (2007) informan que los hombres tienen más probabilidades de aumentar sus creencias de capacidad en la integración de tecnología a través de experiencias de dominio, y las mujeres por medio del aprendizaje vicario. El cuestionario construido no permitió informar en relación a esta diferencia, lo cual es importante considerar en futuras 
investigaciones. Es recomendable desarrollar investigaciones que permitan depurar y redefinir el cuestionario, además, realizar una validación psicométrica que trascienda la validación de expertos en la temática educativa presentada en este artículo (USHER; PAJARES, 2008).

\section{Conclusiones}

La autoeficacia ha demostrado ser un predictor fuerte de las acciones que los futuros profesores proyectan para el ejercicio profesional. Conforme se mencionó anteriormente, creencias positivas implican mayor planeación, esfuerzo, compromiso y persistencia en los procesos de enseñanza, mientras que creencias negativas, implican obstáculos en el ejercicio profesional. La presente investigación tenía por objetivo informar en qué medida se aumenta la autoeficacia para usar tecnología al enseñar matemáticas en la formación inicial de profesores. Al respecto, se reporta un incremento en los valores de la autoeficacia que permiten informar un aumento estadísticamente significativo.

Los análisis minuciosos de este aumento, estadísticamente significativo en la autoeficacia, permitieron establecer cinco conclusiones relevantes: (1) al considerar el dominio funcional específico a partir de las necesidades de formación en el uso de tecnología, el diseño del curso aumentó la autoeficacia y el cuestionario midió dicho aumento de manera eficiente; (2) la autoeficacia de los futuros profesores es más fuerte en el uso de diferentes tecnologías que en el uso de tecnología en diferentes temáticas; (3) las creencias de capacidad en el uso de tecnología con fines educativos son prerrequisito para iniciar reflexiones más elaboradas en sus usos pedagógicos e integración en actividades del aula; (4) los cursos específicos deben trascender la adquisición de habilidades técnicas a una integración eficiente y consciente en los procesos educativos; por último, (5) factores como el género, la edad o la experiencia profesional no presentaron relación con el puntaje de autoeficacia.

Este estudio descriptivo puede constituirse como la fase inicial de futuras investigaciones que se propongan analizar los demás programas acreditados en Colombia para formar profesores de matemáticas. Al respecto, se proporciona una visión de la autoeficacia de los futuros profesores en el contexto local y las características del curso que influyeron en ella, aspectos relevantes para el diseño de cursos orientados al conocimiento del profesor para el uso de tecnologías en la enseñanza de las matemáticas.

Finalmente, esta evidencia contrasta con la implementación de las reformas curriculares que eliminan los cursos de tecnología en la formación inicial de profesores, al respecto, se concluye que un solo curso es necesario, pero no suficiente, para formar en el uso 
de tecnología al enseñar matemáticas.

\section{Referencias}

ABBITT, J. An investigation of the relationship between self-efficacy beliefs about technology integration adn technological pedagogical content knowledge (TPACK) among preservice teachers. Journal of Digital Learning in Teacher Education, Iowa, v. 27, n. 4, p. 134-143, 2011.

ABBITT, J.; KLETT, M. Identifying influences on attitudes and self-efficacy beliefs towards technology integration among pre-service educators. Electronic Journal for the integration of technology in Education, Idaho, v. 6, p. 28-42, 2007.

AGYEI, D.; VOOGT, J. ICT use in the teaching of mathematics: Implications for professional development of pre-service teachers in Ghana. Education and Information Technologies, New York, v. 16, n. 4, p. 423-439, 2011.

AL-AWIDI, H.; ALGHAZO, I. The effect of student teaching experience on preservice elementary teachers' self-efficacy beliefs for technology integration in the UAE. Educational Technology Resarch and Development, Bloomington, v. 60, n. 5, p. 923-941, 2012.

ALVARENGA, C. E. A. Autoeficácia de professores para utilizarem tecnologias de informática no ensino. 2011. Tese (Doutorado em Educação) - Faculdade de Educação, Universidade Estadual de Campinas, Campinas, 2011.

BANDURA, A. Self-efficacy: Toward a unifying theory of behavioral change. Psychological Review, Los Angeles, v. 84, n. 2, p. 191-215, 1977.

BANDURA, A. Self-Efficacy: Encyclopedia of human behavior. San Diego: Academic Press, 1994.

BANDURA, A. Guide for constructing self-efficacy scales. In: PAJARES, F; URDAN, T. (ed.). Selfefficacy beliefs of adolescents. Greenwich: Information Age Publishing, 2006. p. 307-337.

BANDURA, A. On the Functional Properties of Perceived Self-Efficacy Revisited. Journal of Management, Texas, v. 38, n. 1, p. 9-44, 2012.

BYKER, E.; MICHAEL, S.; POLLY, D.; HANDLER, L. Examining Elementary Education Teachers and Preservice Teachers' Self-Efficacy Related to Technological Pedagogical and Content Knowledge (TPACK). In: HODGES, C. (ed.). Self-Efficacy in Instructional Technology Contexts. Cham: Springer International Publishing, 2018. p. 119-140.

CARVAJAL, S. Competencia digital en la formación de profesores en matemáticas. 2018. Tese (Doutorado em Educação) - Didàctica de les Ciències, les Llengües, les Arts i les Humanitats, Universitat de Barcelona, Barcelona, 2018.

CARMONA-MESA, J. A.; ARIAS-SUÁREZ, J.; VILLA-OCHOA, J. A. Formación inicial de profesores basados en proyectos para el diseño de lecciones STEAM. En: Serna, E. (ed.), Revolución en la Formación y la Capacitación para el Siglo XXI, 2a ed., v. 1. Medellin: Editorial IAI, 2019. p. 483-492.

CARMONA-MESA, J. A.; SALAZAR, J. V.; VILLA-OCHOA, J. A. Uso de calculadoras simples y videojuegos en un curso de formación de profesores. Uni-pluriversidad, Medellín, v. 18, n. 1, 13-24. 2018.

CARMONA-MESA, J. A.; VILLA-OCHOA, J. A. Necesidades de formación en futuros profesores 
para el uso de tecnologías. Resultados de un estudio documental. Paradigma, Maracay, v. 38, n. 1, 169-185. 2017.

CASTRILLÓN-YEPES, A.; CARMONA-MESA, J. A.; VILLA-OCHOA, J. A. Technology Integration in a Course for Prospective Mathematics Teachers. In: ROCHA, Á. et al. (ed.), Information Technology and Systems. Cham: Springer, 2020. p. 501-510

COSTA, E. R.; ASSIS, M. P. Crenças de Autoeficácia na Educação: Revisão Sistemática do Período 2007-2017. Revista E-Curriculum, São Paulo, v. 17, n. 4, p. 1909-1929, 2019.

CRESWELL, J. W. Research Design. Qualitative, quantitative and mixed methods approaches, 4. ed. Thousands Oaks: Sage, 2014.

DIGREGORIO, N.; LISTON, D. Experiencing Technical Difficulties: Teacher Self-Efficacy and Instructional Technology. In: HODGES, C. (ed.). Self-Efficacy in Instructional Technology Contexts. Cham: Springer International Publishing, 2018. p. 103-117.

DOUKAKIS, S.; CHIONIDOU-MOSKOFOGLOU, M.; MANGINA-PHELAN, E.; ROUSSOS, P. Measuring technological and content knowledge of undergraduate primary teachers in mathematics. Communications in Computer and Information Science, Berlin, v. 73, p. 405-410, 2010.

DRIJVERS, P.; BALL, L.; BARZEL, B.; HEID, M.; CAO, Y.; MASCHIETTO, M. Uses of Technology in Lower Secondary Mathematics Education. Cham: Springer International Publishing, 2016.

ESCOBAR-PÉREZ, J.; CUERVO-MARTÍNEZ, A. Validez De Contenido Y Juicio De Expertos: Una Aproximación a Su Utilización. Avances en Medición, Bogotá, v. 6, p. 27-36, 2008.

FINK, A. The Survey handbook. 2. ed. Los Ángeles: SAGE Publications, 2003.

GILLHAM, B. Case Study Research Methods. London/New York: Continuum, 2000.

KIILI, C. Measuring and Supporting Pre-Service Teachers Self-Efficacy Towards Computers, Teaching, and Technology Integration. Journal of Technology and Teacher Education, Waynesville, v. 24, n. 4, p. 443-469, 2016.

KILINÇ, M. A Perceptual Scale for Measurement and Evaluation of Prospective Teachers SelfEfficacy in Education. Ahi Evran Üniversitesi Kırşehir Eğitim Fakültesi Dergisi, Kefad, v. 12, n. 4, p. 81-93, 2011.

LEE, Y.; LEE, J. Enhancing pre-service teachers' self-efficacy beliefs for technology integration through lesson planning practice. Computers \& education, Maryland, v. 73, p. 121-128. 2014.

LILJEDAHL, P.; OESTERLE, S. Teacher Beliefs, Attitudes, and Self-Efficacy in Mathematics Education. In: LERMAN, S. (ed.). Encyclopedia of Mathematics Education. 2014. p. 583-585.

MAMA, M.; HENNESSY, S. Developing a typology of teacher beliefs and practices concerning classroom use of ICT. Computers \& Education, Maryland, v. 68, p. 380-387, 2013.

PARASKEVA, F.; BOUTA, H.; PAPAGIANNI, A. Individual characteristics and computer selfefficacy in secondary education teachers to integrate technology in educational practice. Computers \& Education, Maryland, v. 50, n. 3, p. 1084-1091, 2008.

PAMUK, S.; PEKER, D. Turkish pre-service science and mathematics teachers' computer related selfefficacies, attitudes, and the relationship between these variables. Computers \& Education, 
Maryland, v. 53, n. 2, p. 454-461, 2009.

PRIETO, L. Las creencias de autoeficacia docente del profesorado universitario. 2005. Tesis (Doctorado en Psicopedagogía) - Universidad Pontificia Comillas de Madrid, Madrid, 2005.

PRIETO, L. Autoeficacia del profesor universitario: Eficacia percibida y práctica docente. Ciudad de México: Alfaomega Grupo Edito, 2018.

ROBERTSON, M.; AL-ZAHRANI, A. Self-efficacy and ICT integration into initial teacher education in Saudi Arabia : Matching policy with practice Background context - global trends and regional differences. Australasian Journal of Educational Technology, Queensland, v. 28, n. 7, p. 1136$1151,2012$.

SIDDIQ, F.; SCHERER, R. The relation between teachers' emphasis on the development of students' digital information and communication skills and computer self-efficacy: the moderating roles of age and gender. Large-scale Assessments in Education, Amsterdam, v. 4, n. 1, p. 1-21, 2016.

SHAUGHNESSY, M. An interview with Anita Woolfolk: The educational psychology of teacher efficacy. Educational Psychology Review, Wollongong, v. 16, n. 2, p. 153-176, 2004.

SZKLO, M.; NIETO, J. Epidemiologia Intermedia: Conceptos y Aplicaciones. Madrid: Díaz Santos, 2003.

TEO, T. Examining the relationship between student teachers' self-efficacy beliefs and their intended uses of technology for teacing: A structural equation modelling approach. The Turkish Online Journal of Educaional Technology, Sakarya, v. 8, n. 4, p. 7-16, 2009.

TONDEUR, J.; VAN BRAAK, J.; SANG, G.; VOOGT, J.; FISSER, P.; OTTENBREIT-LEFTWICH, A. Preparing pre-service teachers to integrate technology in education: A synthesis of qualitative evidence. Computers \& Education, Maryland, v. 59, n. 1, p. 134-144, 2012.

USHER, E. L.; PAJARES, F. Sources of Self-Efficacy in School: Critical Review of the Literature and Future Directions. Review of Educational Research, New York, v. 78, n. 4, p. 751-796, 2008.

WANG, L.; ERTMER, A.; NEWBY, J. Increasing preservice teachers' self-efficacy beliefs for technology integration. Journal of Research on Technology in Education, London, v. 36, n. 3, p. 231-250, 2004.

WILKERSON, T.; EDDY, C.; QUEBEC-FUENTES, S.; SORTO, M. A.; GUPTA, D.; WARD, E. K.; KERSCHEN, K. Development and validation of the algebra teachers' self-efficacy instrument: Assessment of algebra teachers' knowledge and personal teaching efficacy. School Science and Mathematics, Chigago, v. 118, n. 6, p. 206-217, 2018.

ZHOU, G.; XU, J. Adoption of Educational Technology: How Does Gender Matter? International Journal of Teaching and Learning in Higher Education, Wheatley, v. 19, n. 2, p. 140-153, 2007. 